\title{
SOME INTEGRAL CURVATURE ESTIMATES FOR THE RICCI FLOW IN FOUR DIMENSIONS
}

\author{
MILES SIMON
}

\begin{abstract}
We consider solutions $\left(M^{4}, g(t)\right), 0 \leq t<T$, to Ricci flow on compact, four dimensional manifolds without boundary. We prove integral curvature estimates which are valid for any such solution. In the case that the scalar curvature is bounded and $T<\infty$, we show that these estimates imply that the (spatial) integral of the square of the norm of the Riemannian curvature is bounded by a constant independent of time $t$ for all $0 \leq t<T$ and that the space time integral over $M \times[0, T)$ of the fourth power of the norm of the Ricci curvature is bounded.
\end{abstract}

\section{INTRODUCTION}

We consider arbitrary smooth solutions to Ricci flow, $\frac{\partial}{\partial t} g(t)=-2 \operatorname{Rc}(g(t))$ for all $t \in[0, T)$, on closed, four manifolds without boundary. We assume that the scalar curvature satisfies $\mathrm{R}(\cdot, 0)>-1$ at time zero. If this is not the case, then we can always scale the solution by a constant to obtain a new solution satisfying this inequality. The Ricci flow was first introduced and studied by R. Hamilton in HaThree. We show that the following (and other) integral estimates hold.

Theorem 1.1. Let $\left(M^{4}, g(t)\right)_{t \in[0, T)}$ be a smooth solution to Ricci flow on a compact four dimensional manifold $M^{4}$ without boundary and assume that the scalar curvature satisfies $\inf _{M} \mathrm{R}(\cdot, 0)>-1$ at time zero. Then

$$
\begin{gathered}
\int_{M} \frac{|\mathrm{Rc}|^{2}(\cdot, S)}{(\mathrm{R}(\cdot, S)+2)} d \mu_{g(S)}+\int_{0}^{S} \int_{M} \frac{|\mathrm{Rc}|^{4}(\cdot, t)}{(\mathrm{R}(\cdot, t)+2)^{2}} d \mu_{g(t)} d t \\
\leq 2^{2} \pi^{2} \chi\left(e^{64 S}-1\right)+e^{64 S} \int_{M} \frac{|\mathrm{Rc}|^{2}(\cdot, 0)}{(\mathrm{R}(\cdot, 0)+2)} d \mu_{g(0)} \\
\quad+2^{10} e^{64 S} \int_{0}^{S} \int_{M} \mathrm{R}^{2}(\cdot, t) d \mu_{g(t)} d t \\
=: c_{0}(M, g(0), S)+2^{10} e^{64 S} \int_{0}^{S} \int_{M} \mathrm{R}^{2}(\cdot, t) d \mu_{g(t)} d t
\end{gathered}
$$

and

$$
\int_{M}|\operatorname{Rc}|(\cdot, S) d \mu_{g(S)}
$$

Date: November 9, 2018.

2000 Mathematics Subject Classification. 53C44.

Key words and phrases. Ricci flow, scalar curvature.

We gratefully acknowledge the support of SFB TR71 of the DFG (German Research Foundation) and the University of Freiburg, where a part of this work was carried out. 


$$
\leq \operatorname{vol}(M, g(S))+2 c_{0}(M, g(0), S)+2^{11} e^{64 S} \int_{0}^{S} \int_{M} \mathrm{R}^{2}(\cdot, t) d \mu_{g(t)} d t
$$

and

$$
\begin{aligned}
& \int_{0}^{S} \int_{M}|\mathrm{Rc}|^{2} d \mu_{g(t)} d t \\
& \quad \leq \int_{0}^{S} \operatorname{vol}(M, g(t)) d t+2^{3} c_{0}(M, g(0), S)+2^{13} e^{64 S} \int_{0}^{S} \int_{M} \mathrm{R}^{2}(\cdot, t) d \mu_{g(t)} d t
\end{aligned}
$$

and

$$
\begin{aligned}
& \int_{0}^{S} \int_{M}|\mathrm{Rm}|^{2} d \mu_{g(t)} d t \\
& \quad \leq 4 \int_{0}^{S} \operatorname{vol}(M, g(t)) d t+2^{5}\left(c_{0}(M, g(0), S)+\pi^{2} \chi S\right) \\
& \quad+2^{15} e^{64 S} \int_{0}^{S} \int_{M} \mathrm{R}^{2}(\cdot, t) d \mu_{g(t)} d t
\end{aligned}
$$

for all $0 \leq S<T$, where $\chi=\chi(M)$ is the Euler-characteristic of $M$, and

$$
c_{0}(M, g(0), S):=2^{2} \pi^{2} \chi\left(e^{64 S}-1\right)+e^{64 S} \int_{M} \frac{|\mathrm{Rc}|^{2}(\cdot, 0)}{(\mathrm{R}(\cdot, 0)+2)} d \mu_{g(0)}
$$

is defined in the statement above.

In the case that the scalar curvature is positive everywhere, a minor modification of the proof of these estimates leads to similar estimates which don't contain volume terms: see Theorem 3.4 .

In the remainder of the paper we consider the special case that $T<\infty$ and that the solution has bounded scalar curvature, $\sup _{M^{4} \times[0, T)}|\mathrm{R}| \leq 1$. In this case we see, that the above estimates (and the proofs thereof) imply

$$
\begin{aligned}
& \sup _{t \in[0, T)} \int_{M}|\operatorname{Riem}(\cdot, t)|^{2} d \mu_{g(t)} \leq c_{1}<\infty \\
& \int_{0}^{T} \int_{M}|\operatorname{Rc}|^{4}(\cdot, t) d \mu_{g(t)} d t \leq c_{2}<\infty
\end{aligned}
$$

for explicit constants $c_{1}=c_{1}(M, g(0), T)$ and $c_{2}(M, g(0), T)$ (see Theorem 3.6).

In another paper, BZ, which recently appeared, the authors also consider Ricci flow of four manifolds with bounded scalar curvature. Using different methods, they also independently showed, in this case, that the $L^{2}$ norm of the Riemannian curvature remains bounded as $t \nearrow T$, and they investigate the structure of the limiting space one obtains by letting $t \nearrow T$ : see Theorem 1.8 and Corollary 1.11 of BZ. In a sequel paper, Si], we examine the structure of (possibly) singular limits $\left(X, d_{X}\right):=\lim _{t \nearrow T}(M, d(g(t)))$, where the limit is a Gromov-Hausdorff limit. This limit always exists, and we show that $\left(X, d_{X}\right)$ is a $C^{0}$-Riemannian orbifold, and that it is possible to flow this space for a short time using the orbifold Ricci flow. 


\section{BACKGRound, PREVIOUs RESUlts AND NOTATION}

Here we list some integral curvature estimates that have been shown to hold for the Ricci flow in a general setting. This is by no means an exhaustive list. For more references, we refer to references in the papers we have listed here.

In the paper, HaSurface, the author showed that $\int_{M}(\mathrm{R} \log \mathrm{R})(\cdot, t) d \mu_{g(t)} \leq \int_{M}(\mathrm{R} \log \mathrm{R})(\cdot, 0) d \mu_{g(0)}$ for all $t>0$, for any solution to the normalised Ricci flow $\frac{\partial}{\partial t} g=-2 \mathrm{Rc}+r g$ on a surface (that is, on a two dimensional manifold) which has $\mathrm{R}(\cdot, 0)>0$, where $r(t):=\frac{\int_{M} \mathrm{R}(\cdot, t) d \mu_{g(t)}}{\operatorname{vol}(M, g(t))}$ (see Theorem 7.2 in HaSurface $)$. In ChowI, the author showed that $\int_{0}^{\infty} \int_{M}(\mathrm{R}(\cdot, t)-r(t))^{2} d \mu_{g(t)} d t<\infty$ for any solution to Ricci flow on the sphere.

In the paper [TZ, the authors proved integral curvature estimates for solutions to the normalised Kähler Ricci flow on compact manifolds with bounded diameter and positive scalar curvature (these solutions exist for all time and have bounded scalar curvature and diameter due to a result of Perelman: see $[\mathrm{ST}]$ ). In particular, they show there, that

$\int_{M^{n}}\left(|\operatorname{Riem}|^{2}+|\operatorname{Ricci}|^{4}\right)(\cdot, t) d \mu_{g(t)} \leq \Lambda$ for all $t>0$ for some $\Lambda<\infty$. This estimate is obtained by showing that various integral quantities containing derivatives of the potential function remain bounded as time increases, and using then the ChernWeil Theory (see Section 4 of the paper TZ for details, in particular Lemma 4.2 and Theorem 4.5 there).

As we mentioned in the introduction, in the paper $\mathrm{BZ}$ the authors independently recently showed, that if the scalar curvature is bounded on $[0, T)$ and $M^{n}=M^{4}$ is a four dimensional smooth closed manifold, then the $L^{2}$ integral of the Riemannian curvature remains bounded as $t \nearrow T$, and they investigate the structure of the limiting space one obtains by letting $t \nearrow T$ : see Theorem 1.8 and Corollary 1.11 in $[\mathrm{BZ}$.

\section{Notation:}

We use the Einstein summation convention, and we use the notation of Hamilton HaThree.

For $i \in\{1, \ldots, n\}, \frac{\partial}{\partial x^{i}}$ denotes a coordinate vector, and $d x^{i}$ is the corresponding one form.

$\left(M^{n}, g\right)$ is an $n$-dimensional Riemannian manifold with Riemannian metric $g$.

$g_{i j}=g\left(\frac{\partial}{\partial x^{i}}, \frac{\partial}{\partial x^{i}}\right)$ is the Riemannian metric $g$ with respect to this coordinate system.

$g^{i j}$ is the inverse of the Riemannian metric $\left(g^{i j} g_{i k}=\delta_{j k}\right)$.

$d \mu_{g}$ is the volume form associated to $g$.

$\operatorname{Rm}(g)_{i j k l}={ }^{g} \operatorname{Riem}_{i j k l}=\operatorname{Riem}(g)_{i j k l}=\mathrm{R}_{i j k l}$ is the full Riemannian curvature Tensor.

Weil $(g)_{i j k l}$ is the Weil Tensor.

${ }^{g} \mathrm{Rc}_{i j}=\operatorname{Ricci}_{i j}=\mathrm{R}_{i j}:=g^{k l} \mathrm{R}_{i k j l}$ is the Ricci curvature.

$\mathrm{R}:=\mathrm{R}_{i j k l} g^{i k} g^{j l}$ is the scalar curvature.

${ }^{g} \nabla T=\nabla T$ is the covariant derivative of $T$ with respect to $g$. For example, locally $\nabla_{i} T_{j k}^{s}=(\nabla T)\left(\frac{\partial}{\partial x^{i}}, \frac{\partial}{\partial x^{j}}, \frac{\partial}{\partial x^{k}}, d x^{s}\right)$ (the first index denotes the direction in which the covariant derivative is taken) if locally $T=T_{j k}^{s} d x^{j} \otimes d x^{k} \otimes \frac{\partial}{\partial x^{s}}$.

$|T|={ }^{g}|T|$ is the norm of a tensor with respect to a metric $g$. For example for 
$T=T_{j k}^{s} d x^{j} \otimes d x^{k} \otimes \frac{\partial}{\partial x^{s}} .|T|^{2}=g^{i m} g^{j n} g_{k s} T_{i j}^{s} T_{m n}^{k}$.

Sometimes we make it clearer which Riemannian metric we are considering by including the metric in the definition. For example $\mathrm{R}(h)$ refers to the scalar curvature of the Riemannian metric $h$.

We suppress the $g$ in the notation used for the norm, $|T|={ }^{g}|T|$, and for other quantities, in the case that is is clear from the context which Riemannian metric we are considering.

\section{Integral InEquaLities IN FOUR DimEnsions}

In this paper we consider (unless otherwise stated) smooth families of Riemannian metrics $\left(M^{n}, g(t)\right)_{t \in[0, T)}$ on $n$ dimensional compact, connected manifolds without boundary which solve the Ricci flow equation

$$
\frac{\partial}{\partial t} g(t)=-2 \operatorname{Ricci}(g(t))
$$

for all $t \in[0, T)$. We will mainly be interested in the case that $n=4$.

The following evolution equations hold for the Ricci flow (see HaThree)

$$
\begin{aligned}
& \frac{\partial}{\partial t}|\mathrm{Rc}|^{2}=\Delta|\mathrm{Rc}|^{2}-2|\nabla \mathrm{Rc}|^{2}+4 \mathrm{Rm}^{i k j l} \mathrm{Rc}_{i j} \mathrm{Rc}_{k l} \\
& \frac{\partial}{\partial t} \mathrm{R}=\Delta \mathrm{R}+2|\mathrm{Rc}|^{2} .
\end{aligned}
$$

Applying the maximum principle to the evolution equation for $\mathrm{R}$ above, we obtain the following well known fact: if $\mathrm{R}(x, 0) \geq c$ then $\mathrm{R}(x, t) \geq c$ for all $x \in M$ and all $t \in[0, T)$. In the following, we will assume (unless otherwise stated), that the scalar curvature is bounded from below by -1 for all times: if it is not then we may scale the solution $g(\cdot, t)$ by $\tilde{g}(\cdot, \tilde{t}):=c g\left(\cdot, \frac{\tilde{t}}{c}\right)$, where $c:=\left|\inf _{x \in M} R(x, 0)\right|>0$ to obtain a new solution $(M, \tilde{g}(\tilde{t}))_{t \in[0, \tilde{T})}$, where $\tilde{T}:=c T$, which satisfies $\inf _{M} \tilde{\mathrm{R}}(x, \tilde{t}) \geq-1$ for all $0 \leq \tilde{t}<\tilde{T}$.

This means that the function $f:=\frac{|\mathrm{Rc}|^{2}}{\mathrm{R}+2}$ is well defined.

In the proof of Lemma 3.2 of $\mathrm{CaoX}$ the two evolution equations above were combined to obtain the following evolution equation for $f:=\frac{|\mathrm{Rc}|^{2}}{\mathrm{R}+c}$ at any point in space time where $\mathrm{R}+c>0$ (see also Lemma Knopf], where related evolution inequalities are shown).

$$
\frac{\partial}{\partial t} f=\Delta f-2 \frac{|Z|^{2}}{(\mathrm{R}+c)^{3}}-2 \frac{|\mathrm{Rc}|^{4}}{(\mathrm{R}+c)^{2}}+4 \frac{\mathrm{Rm}(\mathrm{Rc}, \mathrm{Rc})}{(\mathrm{R}+c)}
$$

where $\mathrm{Rm}(\mathrm{Rc}, \mathrm{Rc})$ is the function given locally by $\mathrm{Rm}(\mathrm{Rc}, \mathrm{Rc})=\mathrm{Rm}^{i k j l} \mathrm{Rc}_{i j} \mathrm{Rc}_{k l}$ and $Z:=(\nabla \mathrm{Ricci})(\mathrm{R}+c)-(\nabla \mathrm{R})(\mathrm{Ricci})$ is the tensor given locally by $Z_{k i s}:=$ $\left(\nabla_{k} \mathrm{Rc}\right)_{i s}(\mathrm{R}+c)-\left(\nabla_{k}(\mathrm{R}+c)\right)\left(\mathrm{Rc}_{i s}\right)$. The evolution equation for the integral of $f$ is then given as follows.

Lemma 3.1. Let $\left(M^{n}, g(t)\right)_{t \in[0, T)}$ be a smooth solution to Ricci flow on a four dimensional compact manifold $M^{n}$ without boundary and assume that $\inf _{M} \mathrm{R}(\cdot, 0)>$ 
c. Then

$$
\frac{d}{d t} \int_{M} f d \mu_{g}=\int_{M}\left(-2 \frac{|Z|^{2}}{(\mathrm{R}+c)^{3}}-2 f^{2}+4 \frac{\mathrm{Rm}(\mathrm{Rc}, \mathrm{Rc})}{(\mathrm{R}+c)}-f \mathrm{R}\right) d \mu_{g}
$$

for $f:=\frac{|\mathrm{Rc}|^{2}}{\mathrm{R}+c}$.

Proof. Use the above evolution equation for $f$ with the fact that $\frac{\partial}{\partial t} d \mu_{g}=-\mathrm{R} d \mu_{g}$ (see HaThree for this last fact).

As we mentioned above, there is no great loss of generality in assuming $\inf _{M} \mathrm{R}(\cdot, t) \geq$ -1 for all $t \in[0, T)$, and so we may choose $c=2$ (the special case that $\mathrm{R}>0 \mathrm{ev}$ erywhere, in which case we choose $c=0$, will be handled below separately). We will estimate the last two terms appearing on the right hand side of the integral equality (3.3) using: (i) the Euler characteristic $\chi$, (ii) the good (second) negative term on the right hand side of the above equality

Theorem 3.2. Let $\left(M^{4}, g(t)\right)_{t \in[0, T)}$ be a smooth solution to Ricci flow on a four dimensional compact manifold $M^{4}$ without boundary and assume that $\inf _{M} \mathrm{R}(\cdot, 0)>$ -1 . Then

$$
\frac{d}{d t} \int_{M} f d \mu_{g} \leq 2^{8} \pi^{2} \chi+\int_{M}\left(-f^{2}+64 f+2^{10} \mathrm{R}^{2}\right) d \mu_{g}
$$

for $f:=\frac{|\mathrm{Rc}|^{2}}{\mathrm{R}+2}$.

Proof. We estimate the third term appearing on the right hand side of (3.3) with Young's inequality (remembering that we have now fixed $f=\frac{|\mathrm{Rc}|^{2}}{\mathrm{R}+2}$ in the definition of $f$ ):

$$
\begin{aligned}
4 \frac{\mathrm{Rm}(\mathrm{Rc}, \mathrm{Rc})}{(\mathrm{R}+2)} & \leq \frac{|\mathrm{Rc}|^{4}}{2(\mathrm{R}+2)^{2}}+8|\mathrm{Riem}|^{2} \\
& =\frac{|\mathrm{Rc}|^{4}}{2(\mathrm{R}+2)^{2}}+8\left(|\mathrm{Riem}|^{2}-4|\mathrm{Rc}|^{2}+\mathrm{R}^{2}\right)+32|\mathrm{Rc}|^{2}-8 \mathrm{R}^{2} \\
& =\frac{f^{2}}{2}+8 I+32|\mathrm{Rc}|^{2}-8 \mathrm{R}^{2}
\end{aligned}
$$

where $I=\mid$ Riem $\left.\right|^{2}-4|\mathrm{Rc}|^{2}+\mathrm{R}^{2}$ is the integrand occurring in the generalised Gauss-Bonnet Theorem, and $I$ satisfies

$$
\int_{M} I d \mu_{g}=2^{5} \pi^{2} \chi
$$

where $\chi=\chi(M)$ is the Euler characteristic of $M$ (see notes in Appendix $\mathbb{A}$ ). Note that if $M$ is not oriented, then this formula is correct with $\chi(M):=\frac{1}{2} \chi(\tilde{M})$ where $\tilde{M}$ is the double cover of $M$, which is oriented (see Theorem 13.9 [Lee]), and $\chi(\tilde{M})$ is the Euler-characteristic of $\tilde{M}$. The second last term of the above inequality is $32|\mathrm{Rc}|^{2}=32 f(\mathrm{R}+2)=32 f \mathrm{R}+64 f \leq \frac{f^{2}}{4}+2{ }^{10} \mathrm{R}^{2}+64 f$. Hence

$$
4 \frac{\mathrm{Rm}(\mathrm{Rc}, \mathrm{Rc})}{(\mathrm{R}+2)} \leq \frac{f^{2}}{2}+8 I+\frac{f^{2}}{4}+2^{10} \mathrm{R}^{2}+64 f-8 \mathrm{R}^{2}
$$


Also,

$$
-f \mathrm{R}=\leq \frac{f^{2}}{4}+\mathrm{R}^{2}
$$

Combining these two estimates we obtain

$$
-2 f^{2}+4 \frac{\mathrm{Rm}(\mathrm{Rc}, \mathrm{Rc})}{(\mathrm{R}+2)}-f \mathrm{R} \leq-f^{2}+8 I+2^{10} \mathrm{R}^{2}+64 f .
$$

Using this inequality in the equality (3.3) of the lemma above, we get

$$
\begin{aligned}
\frac{d}{d t} \int_{M} f d \mu_{g} & =\int_{M}\left(-2 \frac{|Z|^{2}}{(\mathrm{R}+2)^{3}}-2 f^{2}+4 \frac{\mathrm{Rm}(\mathrm{Rc}, \mathrm{Rc})}{(\mathrm{R}+2)}-f \mathrm{R}\right) d \mu_{g} \\
& \leq \int_{M}\left(-f^{2}+8 I+2^{10} \mathrm{R}^{2}+64 f\right) d \mu_{g} \\
& =2^{8} \pi^{2} \chi+\int_{M}\left(-f^{2}+64 f+2^{10} \mathrm{R}^{2}\right) d \mu_{g}
\end{aligned}
$$

as required.

Integrating this inequality with respect to time, we obtain

Corollary 3.3. Let $\left(M^{4}, g(t)\right)_{t \in[0, T)}$ be a smooth solution to Ricci flow on a compact four dimensional manifold $M^{4}$ without boundary and assume that $\inf _{M} \mathrm{R}(\cdot, 0)>$ -1 . Then

$$
\begin{aligned}
& \int_{M} \frac{|\mathrm{Rc}|^{2}(\cdot, S)}{(\mathrm{R}(\cdot, S)+2)} d \mu_{g(S)}+\int_{0}^{S} \int_{M} \frac{|\mathrm{Rc}|^{4}(\cdot, t)}{(\mathrm{R}(\cdot, t)+2)^{2}} d \mu_{g(t)} d t \\
& \leq 2^{2} \pi^{2} \chi\left(e^{64 S}-1\right)+e^{64 S} \int_{M} \frac{|\mathrm{Rc}|^{2}(\cdot, 0)}{(\mathrm{R}(\cdot, 0)+2)} d \mu_{g(0)} \\
& \quad+2^{10} e^{64 S} \int_{0}^{S} \int_{M} \mathrm{R}^{2}(\cdot, t) d \mu_{g(t)} d t \\
& =: c_{0}(M, g(0), S)+2^{10} e^{64 S} \int_{0}^{S} \int_{M} \mathrm{R}^{2}(\cdot, t) d \mu_{g(t)} d t
\end{aligned}
$$

$$
\begin{aligned}
& \int_{M}|\operatorname{Rc}|(\cdot, S) d \mu_{g(S)} \\
& \quad \leq \operatorname{vol}(M, g(S))+2 c_{0}(M, g(0), S)+2^{11} e^{64 S} \int_{0}^{S} \int_{M} \mathrm{R}^{2}(\cdot, t) d \mu_{g(t)} d t
\end{aligned}
$$

and

$$
\begin{aligned}
& \int_{0}^{S} \int_{M}|\mathrm{Rc}|^{2} d \mu_{g(t)} d t \\
& \leq \int_{0}^{S} \operatorname{vol}(M, g(t)) d t+2^{3} c_{0}(M, g(0), S)+2^{13} e^{64 S} \int_{0}^{S} \int_{M} \mathrm{R}^{2}(\cdot, t) d \mu_{g(t)} d t
\end{aligned}
$$

and

$$
\begin{aligned}
& \int_{0}^{S} \int_{M}|\operatorname{Rm}|^{2} d \mu_{g(t)} d t \\
& \quad \leq 4 \int_{0}^{S} \operatorname{vol}(M, g(t)) d t+2^{5}\left(c_{0}(M, g(0), S)+\pi^{2} \chi S\right)
\end{aligned}
$$




$$
+2^{15} e^{64 S} \int_{0}^{S} \int_{M} \mathrm{R}^{2}(\cdot, t) d \mu_{g(t)} d t
$$

for all $0 \leq S<T$, where $\chi=\chi(M)$ is the Euler-characteristic of $M$, and

$$
c_{0}(M, g(0), S):=2^{2} \pi^{2} \chi\left(e^{64 S}-1\right)+e^{64 S} \int_{M} \frac{|\mathrm{Rc}|^{2}(\cdot, 0)}{(\mathrm{R}(\cdot, 0)+2)} d \mu_{g(0)}
$$

is defined in the statement above.

Proof. Using the inequality (3.4), we see that

$$
\begin{gathered}
\frac{d}{d t}\left(e^{-64 t} \int_{M} f(\cdot, t) d \mu_{g(t)}\right)+e^{-64 t} \int_{M} f^{2}(\cdot, t) d \mu_{g}(t) \\
\quad \leq e^{-64 t} 2^{8} \pi^{2} \chi+e^{-64 t} \int_{M} 2^{10} \mathrm{R}^{2}(\cdot, t) d \mu_{g}(t)
\end{gathered}
$$

Integrating this inequality from 0 to $S$ implies

$$
\begin{aligned}
e^{-64 S} & \int_{M} f(\cdot, S) d \mu_{g(S)}+e^{-64 S} \int_{0}^{S} \int_{M} f^{2}(\cdot, t) d \mu_{g(t)} d t \\
\leq & e^{-64 S} \int_{M} f(\cdot, S) d \mu_{g(S)}+\int_{0}^{S} e^{-64 t} \int_{M} f^{2}(\cdot, t) d \mu_{g(t)} d t \\
= & \int_{0}^{S}\left(\frac{d}{d t}\left(e^{-64 t} \int_{M} f(\cdot, t) d \mu_{g(t)}\right)+e^{-64 t} \int_{M} f^{2}(\cdot, t) d \mu_{g(t)}\right) d t \\
& +\int_{M} f(\cdot, 0) d \mu_{g(0)} \\
\leq & \int_{0}^{S} e^{-64 t} 2^{8} \pi^{2} \chi d t+\int_{0}^{S} e^{-64 t} \int_{M} 2^{10} \mathrm{R}^{2}(\cdot, t) d \mu_{g}(t) d t+\int_{M} f(\cdot, 0) d \mu_{g(0)} \\
= & -4\left(e^{-64 S}-1\right) \pi^{2} \chi+\int_{0}^{S} e^{-64 t} \int_{M} 2^{10} \mathrm{R}^{2}(\cdot, t) d \mu_{g}(t) d t+\int_{M} f(\cdot, 0) d \mu_{g(0)} \\
\leq & 4\left(1-e^{-64 S}\right) \pi^{2} \chi+\int_{0}^{S} \int_{M} 2^{10} \mathrm{R}^{2}(\cdot, t) d \mu_{g}(t) d t+\int_{M} f(\cdot, 0) d \mu_{g(0)}
\end{aligned}
$$

which, after multiplying by $e^{64 S}$, gives us the first integral inequality (3.10).

The second inequality can be obtained from the first as follows.

$$
\begin{aligned}
|\mathrm{Rc}| & \leq \frac{|\mathrm{Rc}|^{2}}{(\mathrm{R}+1)}+\frac{(\mathrm{R}+1)}{4} \\
& \leq \frac{|\mathrm{Rc}|^{2}}{(\mathrm{R}+1)}+\frac{|\mathrm{R}|}{4}+\frac{1}{4} \\
& \leq \frac{|\mathrm{Rc}|^{2}}{(\mathrm{R}+1)}+\frac{|\mathrm{Rc}|}{2}+\frac{1}{4}
\end{aligned}
$$

in view of the fact that (in four dimensions) $|\mathrm{R}| \leq 2|\mathrm{Rc}|$, and hence

$$
|\mathrm{Rc}| \leq \frac{2|\mathrm{Rc}|^{2}}{(\mathrm{R}+1)}+1
$$

The third inequality can be obtained from the first as follows.

$$
|\mathrm{Rc}|^{2} \leq \frac{4|\mathrm{Rc}|^{4}}{(\mathrm{R}+1)^{2}}+\frac{(\mathrm{R}+1)^{2}}{16}
$$




$$
\begin{aligned}
& \leq \frac{4|\mathrm{Rc}|^{4}}{(\mathrm{R}+1)^{2}}+\frac{|\mathrm{R}|^{2}}{8}+\frac{1}{8} \\
& \leq \frac{4|\mathrm{Rc}|^{4}}{(\mathrm{R}+1)^{2}}+\frac{|\mathrm{Rc}|^{2}}{2}+\frac{1}{8}
\end{aligned}
$$

since $|\mathrm{R}|^{2} \leq 4|\mathrm{Rc}|^{2}$ in four dimensions, and hence

$$
|\mathrm{Rc}|^{2} \leq \frac{8|\mathrm{Rc}|^{4}}{(\mathrm{R}+1)^{2}}+1
$$

The last inequality follows from the third inequality in view of the generalised Gauss-Bonnet theorem.

In the case that $\mathrm{R}>0$ everywhere, we may consider the function $f=\frac{|\mathrm{Rc}|^{2}}{\mathrm{R}}$, that is we choose $c=0$. In this case, some of the terms in the integral inequalities above simplify. In particular, the volume terms don't appear.

Theorem 3.4. Let $\left(M^{4}, g(t)\right)_{t \in[0, T)}$ be a smooth solution to Ricci flow on a compact four dimensional manifold $M^{4}$ without boundary and assume that $\inf _{M} \mathrm{R}(\cdot, 0)>0$. Then

$$
\begin{aligned}
& \int_{M}|\operatorname{Rc}|(\cdot, S) d \mu_{g(S)} \\
& \quad \leq 2 a_{0}(M, g(0), S)+2^{11} e^{64 S} \int_{0}^{S} \int_{M} \mathrm{R}^{2}(\cdot, t) d \mu_{g(t)} d t
\end{aligned}
$$

and

$$
\begin{aligned}
& \int_{0}^{S} \int_{M}|\mathrm{Rc}|^{2} d \mu_{g(t)} d t \\
& \quad \leq 2^{3} a_{0}(M, g(0), S)+2^{13} e^{64 S} \int_{0}^{S} \int_{M} \mathrm{R}^{2}(\cdot, t) d \mu_{g(t)} d t
\end{aligned}
$$

and

$$
\begin{aligned}
& \int_{0}^{S} \int_{M}|\mathrm{Rm}|^{2} d \mu_{g(t)} d t \\
& \quad \leq 2^{5} \pi^{2} \chi S+2^{5} a_{0}(M, g(0), S)+2^{15} e^{64 S} \int_{0}^{S} \int_{M} \mathrm{R}^{2}(\cdot, t) d \mu_{g(t)} d t
\end{aligned}
$$

for all $0 \leq S<T$, where $\chi=\chi(M)$ is the Euler-characteristic of $M$, and

$$
a_{0}(M, g(0), S):=2^{2} \pi^{2} \chi\left(e^{64 S}-1\right)+e^{64 S} \int_{M} \frac{|\mathrm{Rc}|^{2}}{\mathrm{R}}(\cdot, 0) d \mu_{g(0)} .
$$

Proof. We repeat the argument given in the proof of Theorem 3.2, but we use the function $f=\frac{|\mathrm{Rc}|^{2}}{\mathrm{R}}$ in place of $f=\frac{|\mathrm{Rc}|^{2}}{\mathrm{R}+2}$. We use the fact that $|\mathrm{Rc}|=\frac{|\mathrm{Rc}|^{2}}{|\mathrm{Rc}|} \leq 2 \frac{|\mathrm{Rc}|^{2}}{\mathrm{R}}=$ $2 f$ in four dimensions in the last part of the argument to get (3.20) and (3.21). The generalised Gauss-Bonnet theorem implies the last inequality (3.22).

In the rest of this paper we often consider solutions $\left(M^{4}, g(t)\right)_{t \in[0, T)}$ which satisfy the following basic assumptions.

(a) $M^{4}$ is a smooth, compact, connected four dimensional manifold without boundary 
(b) $\left(M^{4}, g(t)\right)_{t \in[0, T)}$ is a smooth solution to the Ricci flow $\frac{\partial}{\partial t} g(t)=-2 \operatorname{Ricci}(g(t))$ for all $t \in[0, T)$

(c) $T<\infty$

(d) $\sup _{M^{4} \times[0, T)}|\mathrm{R}(x, t)| \leq 1$

If instead of $(d)$ we only have $\sup _{M \times[0, T)}|\mathrm{R}(x, t)| \leq K<\infty$ for some constant $1<K<\infty$, then we may rescale the solution $\tilde{g}(\cdot, \tilde{t}):=K g\left(\cdot, \frac{\tilde{t}}{K}\right)$ to obtain a new solution $(M, \tilde{g}(\tilde{t}))_{t \in[0, \tilde{T})}$, where $\tilde{T}:=K T$, which satisfies the basic assumptions.

Note that a solution which satisfies the basic assumptions also satisfies $\mathrm{R}(x, t)+2>$ 0 for all $x \in M$ for all $t \in[0, T)$ and hence $f:=\frac{|\mathrm{Rc}|^{2}}{\mathrm{R}+2}$ is a well defined function.

For solutions satisfying the basic assumptions, a slight modification of the above arguments leads to the following.

Theorem 3.5. Let $\left(M^{4}, g(t)\right)_{t \in[0, T)}$ be a solution to Ricci flow satisfying the basic assumptions. Then

$$
\frac{d}{d t} \int_{M} f d \mu_{g} \leq 128 \pi^{2} \chi+\int_{M}\left(-f^{2}+50 f\right) d \mu_{g}
$$

for $f:=\frac{|\mathrm{Rc}|^{2}}{\mathrm{R}+2}$, where $\chi=\chi(M)$ is the Euler characteristic of $M$.

Proof. Using almost the same argument given at the beginning of the proof of Theorem 3.2, we see that

$$
4 \frac{\mathrm{Rm}(\mathrm{Rc}, \mathrm{Rc})}{(\mathrm{R}+2)} \leq f^{2}+4 I+16|\mathrm{Rc}|^{2},
$$

where $I=\mid$ Riem $\left.\right|^{2}-4|\mathrm{Rc}|^{2}+\mathrm{R}^{2}$ is the integrand occurring in the generalised GaussBonnet Theorem. The last term of the above inequality is $16|\mathrm{Rc}|^{2}=16 f(\mathrm{R}+2) \leq$ $48 f$ since $\mathrm{R} \leq 1$. Hence

$$
4 \frac{\mathrm{Rm}(\mathrm{Rc}, \mathrm{Rc})}{(\mathrm{R}+2)} \leq f^{2}+4 I+48 f
$$

Also,

$$
-f \mathrm{R}=-f(\mathrm{R}+2)+2 f \leq 2 f .
$$

Combining these two estimates we obtain

$$
-2 f^{2}+4 \frac{\mathrm{Rm}(\mathrm{Rc}, \mathrm{Rc})}{(\mathrm{R}+2)}-f \mathrm{R} \leq-f^{2}+4 I+50 f .
$$

Using this inequality in the equality (3.3), we get

$$
\begin{aligned}
\frac{d}{d t} \int_{M} f d \mu_{g} & =\int_{M}\left(-2 \frac{|Z|^{2}}{(\mathrm{R}+2)^{3}}-2 f^{2}+4 \frac{\mathrm{Rm}(\mathrm{Rc}, \mathrm{Rc})}{(\mathrm{R}+2)}-f \mathrm{R}\right) d \mu_{g} \\
& \leq \int_{M}\left(-f^{2}+4 I+50 f\right) d \mu_{g} \\
& =128 \pi^{2} \chi+\int_{M}\left(-f^{2}+50 f\right) d \mu_{g}
\end{aligned}
$$

as required. 
Theorem 3.6. Let $\left(M^{4}, g(t)\right)_{t \in[0, T)}$ be a smooth solution to Ricci flow satisfying the basic assumptions. Then we have the following estimates:

$$
\begin{aligned}
& \int_{M}|\operatorname{Rc}|^{2}(\cdot, t) d \mu_{g(t)} \leq b(g(0), t) \quad \forall t \in[0, T) \\
& \int_{M}|\operatorname{Riem}|^{2}(\cdot, t) \quad d \mu_{g(t)} \leq 32 \pi^{2} \chi+4 b(g(0), t) \quad \forall t \in[0, T) \\
& \int_{0}^{t} \int_{M}|\mathrm{Rc}|^{4}(\cdot, t) d \mu_{g(t)} d t \leq b(g(0), t) \quad \forall t \in[0, T] \\
& \int_{S}^{T} \int_{M}|\operatorname{Rc}|^{p}(\cdot, t) d \mu_{g(t)} d t \\
& \leq\left(\mid b(g(0), T \mid)^{\frac{p}{4}} e^{\frac{(4-p) T}{4}}\left(\operatorname{vol}(M, g(0))^{\frac{(4-p)}{4}}|T-S|^{\frac{(4-p)}{4}} \rightarrow 0 \text { as } S \nearrow T\right.\right.
\end{aligned}
$$

for all $0<p<4$, where

$$
b(g(0), t) \quad:=50 e^{50 t} \int_{M}|\operatorname{Rc}|^{2}(\cdot, 0) d \mu_{g(0)}+128 \pi^{2} \chi\left(e^{50 t}-1\right)
$$

and $\chi$ is the Euler characteristic.

Remark 3.7. In the above inequalities, we may estimate $b(g(0), t)$ and $|b(g(0), t)|$ by $b(g(0), t) \leq \mid b\left(\left.g(0, t)\left|\leq c(g(0), T):=50 e^{50 T} \int_{M}\right| \operatorname{Rc}\right|^{2}(\cdot, 0) d \mu_{g(0)}+128 \pi^{2}|\chi| e^{50 T}\right.$

Remark 3.8. Note that $b(h, s):=b(\tilde{h}, s)$ if $\tilde{h}=c h$ and $c>0, s>0$ are arbitrary, in view of the fact that $\int_{M}|\operatorname{Rc}(h)|^{2} d \mu_{h}=\int_{M}|\tilde{\operatorname{Rc}}(\tilde{h})|^{2} d \mu_{\tilde{h}}$ in four dimensions, as one readily verifies using the definition of Riemannian curvature (see for example the definition given in Section 2 of HaThree).

Remark 3.9. In a recent paper, $[\mathrm{BZ}$, the authors independently showed (among other things) that $\sup _{t \in[0, T)} \int_{M}|\mathrm{Rc}|^{2}(\cdot, t) d \mu_{g(t)}<\infty$ and

$\sup _{t \in[0, T)} \int_{M}|\operatorname{Rm}|^{2}(\cdot, t) d \mu_{g(t)}<\infty$ if $\left(M^{4}, g(t)\right)_{t \in[0, T)}$ is a solution to Ricci flow satisfying the basic assumptions. Their method uses estimates on the heat kernel, which are also proved in their paper, and their method is different from the method presented here.

Proof. From Theorem (3.5) above we have

$$
\frac{d}{d t} \int_{M} f d \mu_{g} \leq 128 \pi^{2} \chi+\int_{M}\left(-f^{2}+50 f\right) d \mu_{g}
$$

and hence

$$
\frac{d}{d s}\left(e^{-50 s} \int_{M} f(\cdot, s) d \mu_{g(s)}\right) \leq e^{-50 s} 128 \pi^{2} \chi-e^{-50 s} \int_{M} f^{2} d \mu_{g(s)}
$$

Integrating both sides of this inequality in time from 0 to $t<T$, we get

$$
\begin{gathered}
e^{-50 t} \int_{0}^{t} \int_{M} f^{2} d \mu_{g(s)} d s+e^{-50 t} \int_{M} f d \mu_{g(t)} \\
\leq \int_{M} f(\cdot, 0) d \mu_{g}(0)+\frac{128}{50} \pi^{2} \chi\left(1-e^{-50 t}\right) .
\end{gathered}
$$

Using the definition of $f$ and the fact that $1 \leq(\mathrm{R}+2) \leq 3$ we see that

$$
\frac{|\mathrm{Rc}|^{2}}{3} \leq f=\frac{|\mathrm{Rc}|^{2}}{\mathrm{R}+2} \leq|\mathrm{Rc}|^{2}
$$


and hence, using this in (3.35), we see that

$$
\begin{aligned}
& \frac{e^{-50 t}}{50} \int_{0}^{t} \int_{M}|\mathrm{Rc}|^{4} d \mu_{g(s)} d s+\frac{e^{-50 t}}{50} \int_{M}|\mathrm{Rc}|^{2} d \mu_{g(t)} \\
& \quad \leq e^{-50 t} \int_{0}^{t} \int_{M} f^{2} d \mu_{g(s)} d s+e^{-50 t} \int_{M} f d \mu_{g(t)} \\
& \leq \int_{M} f(\cdot, 0) d \mu_{g}(0)+\frac{128}{50} \pi^{2} \chi\left(1-e^{-50 t}\right) \\
& \leq \int_{M}|\mathrm{Rc}|^{2}(\cdot, 0) d \mu_{g(0)}+\frac{128}{50} \pi^{2} \chi\left(1-e^{-50 t}\right)
\end{aligned}
$$

This gives us the first (3.29) and third (3.31) estimate.

The second inequality, (3.30), follows from the first inequality and the generalised Gauss-Bonnet Theorem:

$$
\int_{M}|\operatorname{Riem}|^{2} d \mu_{g}=32 \pi^{2} \chi+\int_{M}\left(4|\mathrm{Rc}|^{2}-\mathrm{R}^{2}\right) d \mu_{g} \leq 32 \pi^{2} \chi+4 b(g(0), t),
$$

as required.

The equation for the evolution of the volume is (see Section 3 of HaThree) $\frac{d}{d t} \operatorname{vol}(M, g(t))=-\int_{M} \mathrm{R} d \mu_{g(t)}$, and hence using $|\mathrm{R}| \leq 1$ we see that $-\operatorname{vol}(M, g(t)) \leq$ $\frac{d}{d t} \operatorname{vol}(M, g(t)) \leq \operatorname{vol}(M, g(t))$. Integrating this inequality from 0 to $t$ we see that $e^{-T} \operatorname{vol}(M, g(0)) \leq \operatorname{vol}(M, g(t)) \leq e^{T} \operatorname{vol}(M, g(0))$. Using Hölder's inequality and these volume bounds we get for $p<4$ and $S<R<T$

$$
\begin{aligned}
\int_{S}^{R} \int_{M}|\operatorname{Rc}|^{p}(\cdot, l) d \mu_{g(l)} d l & \leq\left(\int_{S}^{R} \int_{M}|\operatorname{Rc}|^{4} d \mu_{g(l)} d l\right)^{p / 4}\left(\int_{S}^{R} \int_{M} d \mu_{g(l)} d l\right)^{1 / q} \\
& \leq\left(\int_{0}^{T} \int_{M}|\operatorname{Rc}|^{4} d \mu_{g(l)} d l\right)^{p / 4}|S-R|^{\frac{1}{q}} e^{\frac{T}{q}}\left(\operatorname{vol}(M, g(0))^{\frac{1}{q}}\right. \\
& \leq|b(g(0), T)|^{\frac{p}{4}} e^{\frac{T}{q}}\left(\operatorname{vol}(M, g(0))^{\frac{1}{q}}|S-R|^{\frac{1}{q}}\right.
\end{aligned}
$$

where $\frac{1}{q}=1-\frac{p}{4}=\frac{(4-p)}{4}$. This implies the fourth inequality, (3.32) above.

This completes the proof.

In four dimensions, $\int_{M}|\mathrm{Rc}|^{2} d \mu_{g}$ and $\int_{M} \mid$ Riem $\left.\right|^{2} d \mu_{g}$ are scale invariant quantities: if $\tilde{g}=c g, c>0$, then $\int_{M}|\tilde{\mathrm{Rc}}|^{2} d \mu_{\tilde{g}}=\int_{M}|\mathrm{Rc}|^{2} d \mu_{g}$ and $\int_{M}|\mathrm{Rm}|^{2} d \mu_{g}=$ $\int_{M}|\tilde{\mathrm{Rm}}|^{2} d \mu_{\tilde{g}}$, as can be readily verified using the definition of Riemannian curvature, as we mentioned above.

These facts help us to obtain inequalities for scaled solutions, as explained below in the proof of the following theorem.

Theorem 3.10. Let $\left(M^{4}, g(t)\right)_{t \in[0, T)}$ be a smooth solution to Ricci flow satisfying the basic assumptions. Let $\tilde{g}(\cdot, \tilde{t}):=c g\left(\cdot, \frac{\tilde{t}}{c}\right)$ for $0 \leq \tilde{t} \leq \tilde{T}:=c T$ for any constant $c>0$, and let $0 \leq \tilde{R}<\tilde{S} \leq \tilde{T}$. Then

$$
\begin{aligned}
& \int_{M}|\tilde{\mathrm{Rc}}|^{2}(\cdot, \tilde{t}) d \mu_{g(\tilde{t})} \leq b(g(0), t) \\
& \int_{M}|\operatorname{Riem}|^{2}(\cdot, \tilde{t}) \quad d \mu_{g(\tilde{t})} \leq 32 \pi^{2} \chi+4 b(g(0), t)
\end{aligned}
$$


for all $\tilde{t} \in[0, \tilde{T}]$, where $t:=\frac{\tilde{t}}{c}$. In the case that we additionally assume $c \geq 1$, then we also have

$$
\int_{\tilde{R}}^{\tilde{S}} \int_{M}|\tilde{\operatorname{Rc}}|^{4}(\cdot, \tilde{l}) d \mu_{g(\tilde{l})} d \tilde{l} \leq 50 e^{50 \tilde{L}} b(g(0), R)+128 \pi^{2} \chi\left(e^{50 \tilde{L}}-1\right)
$$

where $\tilde{L}=\tilde{S}-\tilde{R}, b(g(0), t)$ is defined above in (3.33) and $\tilde{t}=c t, R:=\frac{\tilde{R}}{c}, S=\frac{\tilde{S}}{c}$.

Remark 3.11. Note that $b(g(0), t)$ is not equal to $b(\tilde{g}(0), \tilde{t}))$ in general: for fixed $t \in[0, T)$, the quantity $b(\tilde{g}(0), \tilde{t}) \rightarrow \infty$ as $c \rightarrow \infty$ for $\tilde{t}:=c t$, if for example $\chi>0$. As we mentioned above, we do have however $b(g(0), t)=b(\tilde{g}(0), t)$ for all $t>0$.

Proof. The first two inequalities follow from the fact that the left hand side of the inequality is scale invariant (see the explanation given just before the statement of this theorem). Now we consider the case that $c \geq 1$. Define $(M, h(t))_{t \in[0, \tilde{L}:=\tilde{S}-\tilde{R})}$ to be $h(s):=\tilde{g}(\cdot, \tilde{R}+s)$. Then $(M, h(s))_{s \in[0, \tilde{L})}$ is a solution satisfying the basic assumptions, and so we may apply the results above to obtain

$$
\begin{aligned}
\int_{\tilde{R}}^{\tilde{S}} \int_{M}|\tilde{\mathrm{Rc}}|^{4}(\cdot, \tilde{t}) d \mu_{\tilde{g}(\tilde{t})} d \tilde{t} & =\int_{0}^{\tilde{L}} \int_{M}|\operatorname{Rc}|^{4}(\cdot, t) d \mu_{h(t)} d t \\
& \leq b(h(0), \tilde{L}) \\
& =50 e^{50 \tilde{L}} \int_{M}|\operatorname{Rc}|^{2}(\cdot, 0) d \mu_{h}(0)+128 \pi^{2} \chi\left(e^{50 \tilde{L}}-1\right) \\
& =50 e^{50 \tilde{L}} \int_{M}|\tilde{\operatorname{Rc}}|^{2}(\cdot, \tilde{R}) d \mu_{\tilde{g}(\tilde{R})}+128 \pi^{2} \chi\left(e^{50 \tilde{L}}-1\right) \\
& \leq 50 e^{50 \tilde{L}} b(g(0), R)+128 \pi^{2} \chi\left(e^{50 \tilde{L}}-1\right)
\end{aligned}
$$

where the last inequality here follows in view of the first (scale invariant) inequality (3.40). This finishes the proof.

The following corollaries are obtained using the above integral estimates.

Corollary 3.12. Let $\left(M^{4}, g(t)\right)_{t \in[0, T)}$ be a smooth solution to Ricci flow on a closed four manifold $M$ satisfying the basic estimates with $T<\infty$. Then

$$
\begin{aligned}
& \int_{0}^{T} \int_{M}|\nabla \mathrm{Rc}|^{2} d \mu_{g(t)} d t \\
& \quad \leq B(g(0), T) \\
& \quad:=\int_{M}|\operatorname{Rc}(\cdot, 0)|^{2} d \mu_{g(0)}+b(g(0), T)+2^{9} \pi^{2} \chi T \\
& \quad+2^{6}\left(\left(e^{50 T}-1\right) \int_{M}|\operatorname{Rc}(\cdot, 0)|^{2} d \mu_{g(0)}+128 \pi^{2} \chi\left(\frac{e^{50 T}}{50}-\frac{1}{50}-T\right)\right)
\end{aligned}
$$

Remark 3.13. Note that $B(h, s)=B(c h, s)$ for all $c>0$, for all $s>0$, since this is true for $b(h, s)$, and $\int_{M}|\mathrm{Rc}|^{2} d \mu_{g}$ is invariant under scaling (as we explained above).

Proof. As mentioned above, see (3.1), the evolution equation for $|\mathrm{Rc}|^{2}$ is

$$
\frac{\partial}{\partial t}|\mathrm{Rc}|^{2}=\Delta|\mathrm{Rc}|^{2}-2|\nabla \mathrm{Rc}|^{2}+4 \mathrm{Rm}(\mathrm{Rc}, \mathrm{Rc}) .
$$


Integrating this over space and time from 0 to $T$ we get

$$
\begin{aligned}
& \int_{0}^{T} \int_{M}|\nabla \mathrm{Rc}|^{2}(\cdot, t) \mu_{g(t)} d t \\
& \leq \int_{M}|\operatorname{Rc}|^{2}(\cdot, 0) d \mu_{g(0)}+\int_{0}^{T} \int_{M} 4|\operatorname{Rm}(\mathrm{Rc}, \mathrm{Rc})|(\cdot, t) d \mu_{g(t)} d t \\
& \leq \int_{M}|\operatorname{Rc}|^{2}(\cdot, 0) d \mu_{g(0)}+\int_{0}^{T} \int_{M}|\operatorname{Rc}|^{4}(\cdot, t) d \mu_{g(t)} d t \\
& \quad+\int_{0}^{T} \int_{M} 16|\operatorname{Rm}|^{2}(\cdot, t) d \mu_{g(t)} d t
\end{aligned}
$$

Now we use the inequalities (3.30) and (3.31) to get

$$
\begin{aligned}
& \int_{0}^{T} \int_{M}|\nabla \mathrm{Rc}|^{2} d \mu_{g(t)} d t \\
& \leq \int_{M}|\operatorname{Rc}(\cdot, 0)|^{2} d \mu_{g(0)}+b(g(0), T)+16 \int_{0}^{T}\left(32 \pi^{2} \chi+4 b(g(0), t)\right) d t \\
& =\int_{M}|\operatorname{Rc}(\cdot, 0)|^{2} d \mu_{g(0)}+b(g(0), T)+16 \cdot 32 \pi^{2} \chi T+16 \cdot 4 \cdot \int_{0}^{T} b_{t} d t \\
& =\int_{M}|\operatorname{Rc}(\cdot, 0)|^{2} d \mu_{g(0)}+b(g(0), T)+2^{9} \pi^{2} \chi T \\
& +2^{6}\left(\left(e^{50 T}-1\right) \int_{M}|\operatorname{Rc}(\cdot, 0)|^{2} d \mu_{g(0)}+128 \pi^{2} \chi\left(\frac{e^{50 T}}{50}-\frac{1}{50}-T\right)\right) \\
& =: B(g(0), T)
\end{aligned}
$$

as required

Corollary 3.14. Let $\left(M^{4}, g(t)\right)_{t \in[0, T)}$ be a smooth solution to Ricci flow on a closed four manifold $M$ with $T<\infty$. Let $K:=\sup _{M \times[0, T)}|\mathrm{R}|<\infty$ and assume $K \geq 1$. Then we have the following estimates:

$$
\begin{aligned}
& \int_{M}|\operatorname{Rc}|^{2}(\cdot, t) d \mu_{g(t)} \leq b(g(0), K t) \quad \forall t \in[0, T) \\
\forall t \in[0, T) \quad & \int_{M}|\operatorname{Riem}|^{2}(\cdot, t) \quad d \mu_{g(t)} \leq 32 \pi^{2} \chi+4 b(g(0), K t) \\
& \int_{0}^{T} \int_{M}|\operatorname{Rc}|^{4}(\cdot, t) d \mu_{g(t)} d t \leq K b(g(0), K T) \\
& \int_{0}^{T} \int_{M}|\nabla \mathrm{Rc}|^{2} d \mu_{g(t)} d t \leq K^{3} B(g(0), K T)
\end{aligned}
$$

where $b(g(0), s), B(g(0), s)$ are as defined in (3.33) and (3.43) $(s \in[0, \infty)$ is arbitrary in the definition).

Remark 3.15. If $K \leq 1$, then we may estimate the integrals involved using Theorem 3.6 and Corollary 3.12

Proof. Set $\tilde{g}(\cdot, \tilde{t}):=K g\left(\cdot, \frac{\tilde{t}}{K}\right)$ for $\tilde{t} \in[0, T K=: \tilde{T}]$. The result now follows from Theorem [3.6 and Corollary 3.12 applied to the solution $\tilde{g}(\cdot, \tilde{t})_{t \in[0, \tilde{T})}$ in view of the fact that $\tilde{T}=T K$ and the identities $\int_{M}|\operatorname{Rc}|^{2}(\cdot, t) d \mu_{g(t)}=\int_{M}|\tilde{R c}|^{2}(\cdot, \tilde{t}) d \mu_{\tilde{g}(\tilde{t})}$, $\int_{M}|\operatorname{Rm}|^{2}(\cdot, t) d \mu_{g(t)}=\int_{M}|\tilde{\operatorname{Rm}}|^{2}(\cdot, \tilde{t}) d \mu_{\tilde{g}(\tilde{t})}$, 
$\int_{0}^{T} \int_{M}|\mathrm{Rc}|^{4} d \mu_{g(t)} d t=K \int_{0}^{\tilde{T}} \int_{M}|\tilde{\mathrm{Rc}}|^{4} d \mu_{\tilde{g}(\tilde{t})} d \tilde{t}$, $\int_{0}^{T} \int_{M}|\nabla \mathrm{Rc}|^{4} d \mu_{g(t)} d t=K^{3} \int_{0}^{\tilde{T}} \int_{M}|\tilde{\nabla} \tilde{\mathrm{Rc}}|^{4} d \mu_{\tilde{g}(\tilde{t})} d \tilde{t}$, which all follow from the scaling, that is $\tilde{g}(\cdot, \tilde{t})=K g\left(\cdot, \frac{\tilde{t}}{K}\right)$, and the fact that $B(g(0), s)=B(\tilde{g}(0), s), b(g(0), s)=$ $b(\tilde{g}(0), s)$ for all $s>0$, as we mentioned above.

\section{Appendix A. Notes on the Euler characteristic}

In the following, we assume that $\left(M^{n}, g\right)$ is an oriented smooth compact Riemannian manifold without boundary (unless otherwise stated). The Pfaffian is a second order polynomial obtained using the curvature operator: see section 3 in [BG], for example, for a definition. In the case that the Riemannian manifold $\left(M^{4}, g\right)$ we consider has dimension four, the Pfaffian Pf may be written as Pf $=c\left(\mid\right.$ Riem $\left.\right|^{2}-$ $\left.4|\mathrm{Rc}|^{2}+\mathrm{R}^{2}\right) \operatorname{vol}_{g}$ where $\operatorname{vol}_{g}$ is the Riemannian volume form. This may be seen by using the Formula 4.1 and Corollary 4.1 in BG] ( using the orthonormal basis $X_{1}, X_{2}, X_{3}, X_{4}$ given at $p \in M$ coming from Corollary 4.1 in BG] we can calculate $\left(|\mathrm{Riem}|^{2}-4|\mathrm{Rc}|^{2}+\mathrm{R}^{2}\right.$ ) $\operatorname{vol}_{g}$ and we see that it has the same value (up to a constant) of $\mathrm{Pf}$ at $p$ given in the Formula 4.1 of $[\mathrm{BG}]$ ). It is known ([AW], Chern]), that the generalised Gauss-Bonnet formula $c(n) \int_{M} \mathrm{Pf}=\chi(M)$ holds: for an intrinsic explanation using modern day terminology, see $[\mathrm{Br}$. One may choose the generic vector field $Y$ occurring in the explanation of Bryant to be $\nabla f$, where $f: M \rightarrow \mathbb{R}$ is a morse-function (see section 6 in [Mi] to see that such functions exist). The Euler characteristic is $\chi(M):=c_{0}-c_{1}+c_{2}-c_{3}+\ldots+(-1)^{n} c_{n}=b_{0}-b_{1}+b_{2}-b_{3}+\ldots+$ $(-1)^{n} b_{n}$, where here, $b_{i}$ is the $i$ th Betti-number, and $c_{i}$ is the number of critical values of degree $i$ : see Theorem 5.2 in [Mi]. So we have $a \int_{M}\left(|\mathrm{Riem}|^{2}-4|\mathrm{Rc}|^{2}+\right.$ $\left.\mathrm{R}^{2}\right) \operatorname{vol}_{g}=\chi(M)$ for some constant $a$. To see that the constant $a$ in this formula is $\frac{1}{32 \pi^{2}}$, calculate the left and right hand side of this formula in the case that $(M, g)$ is the standard sphere with sectional curvature equal to one everywhere.

\section{REFERENCES}

[AW] Allendoerfer, C., Weil, A. The Gauss-Bonnet theorem for Riemannian polyhedra, Trans. Amer. Math. Soc., 53 (1943), 101-129.

[BG] Bishop, R.L., Goldberg, S. I. Some implications of the generalized Gauss-Bonnet

[BZ] Bamler, R., Zhang, Q., Heat kernel and curvature bounds in Ricci flows with bounded scalar curvature arXiv:1501.01291 (2015)

[Br] Bryant, R. A question on Generalized Gauss-Bonnet Theorem, mathoverflow.net/questions/84521/a-question-on-generalized-gauss-bonnet-theorem

[CaoX] Cao, X.-, Curvature Pinching Estimate and Singularities of the Ricci Flow, Comm. Anal. Geom., Vol. 19 (5): 975-990, (2011).

[ChowI] Chow, B., The Ricci flow on the two sphere, J. Differential Geom. Volume 33, Number 2 (1991), 325-334.

[Chern] Chern, S-S., A Simple Intrinsic Proof of the Gauss-Bonnet Formula for Closed Riemannian Manifolds, Annals of Mathematics, Second Series, Vol. 45, No. 4 (1944), pp. 747-752

[HaThree] Hamilton,R.S., Three-manifolds with positive Ricci curvature, Journal of Differential Geometry 17 (2):255-306(1982)

[HaSurface] Hamilton, R.S., Ricci flow on surfaces, Mathematics and general relativity, Cont. Math. 71 (1988) 237-261.

[Knopf] Knopf, D., Estimating the trace-free Ricci tensor in Ricci flow, Proc. Amer. Math. Soc. 137 (2009), no. 9, 3099-3103.

[Lee] Lee, J., Introduction to smooth manifolds, Graduate texts in Math., Springer (2002) 
[Mi] , Milnor, J., Morse Theory, Princeton University Press, (1963)

[Si] Simon, M. Extending four dimensional Ricci flows with bounded scalar curvature, Arxiv Preprint

[ST] Sesum, N., Tian, G. Bounding scalar curvature and diameter along the Kaehler Ricci flow (after Perelman), Journal of the Inst. of Mathematics of Jussieu, Vol. 7, 03, (2008) pp. 575-587

[TZ] Tian, G., Zheng, Z. Regularity of Kaehler Ricci flows on manifolds, arXiv:1310.5897

Miles Simon: Otto von Guericke University, Magdeburg, iAn, Universitätsplatz 2, Magdeburg 39104, Germany

E-mail address: miles point simon at ovgu point de 